\title{
Acute Oral Potassium Overdose: The Role of Hemodialysis
}

\author{
George M. Bosse • Melissa A. Platt • \\ Stephen D. Anderson • Michael W. Presley
}

Published online: 19 August 2010

(C) American College of Medical Toxicology 2010

\begin{abstract}
Introduction Hyperkalemia is a common condition, particularly in the setting of renal dysfunction. Hyperkalemia due to intentional oral potassium overdose is not commonly reported.

Case Report We present a case of acute intentional potassium overdose in a patient with normal renal function resulting in significant hyperkalemia, with a maximum serum potassium concentration of $11 \mathrm{mEq} / \mathrm{L}$. Despite an initial course complicated by various unstable cardiac rhythms, including ventricular tachycardia, ventricular fibrillation, and pulseless electrical activity, the patient was discharged from the hospital neurologically intact. Treatment for hyperkalemia included hemodialysis.

Discussion The role of dialysis in potassium overdose is poorly defined.

Conclusion Based on this case and a review of the medical literature, we recommend hemodialysis for cases of potassium overdose with hemodynamic instability and significantly
\end{abstract}

Notes: There was no outside funding of any kind used for this study.

G. M. Bosse $(\bowtie)$

Department of Emergency Medicine, University of Louisville,

530 S. Jackson Street,

Louisville, KY 40202, USA

e-mail: george.bosse@nortonhealthcare.org

M. A. Platt

University of Louisville,

Louisville, KY, USA

S. D. Anderson

Baptist Hospital,

Nashville, TN, USA

M. W. Presley

Frankfort Regional Medical Center,

Frankfort, KY, USA elevated serum potassium concentrations that do not respond promptly to medical therapy. Hemodialysis should also be considered in cases with underlying renal dysfunction.

Keywords Hyperkalemia $\cdot$ Potassium $\cdot$ Overdose $\cdot$ Dialysis

\section{Introduction}

Hyperkalemia with cardiac arrest due to acute intentional oral potassium overdose is uncommon. Previously described cases demonstrate inconsistent management approaches. Potassium excretion in such cases would be expected to be rapid in the setting of normal function.

We describe a case of potassium overdose resulting in significant hyperkalemia, with a potassium concentration as high as $11 \mathrm{mEq} / \mathrm{L}$. The patient presented with a "sine wave" cardiac rhythm, and the initial course was complicated by various other unstable rhythms including pulseless electrical activity, ventricular tachycardia, and ventricular fibrillation. Treatment included both pharmacologic management of hyperkalemia and hemodialysis. The patient was discharged from the hospital neurologically intact after a 7-day course. This is unusual considering that the initial period of cardiovascular instability with dysrhythmias lasted approximately $2 \mathrm{~h}$, followed by persistent hypotension requiring vasopressor support for an additional 5 days.

The patient had also ingested lisinopril, an angiotensinconverting enzyme (ACE) inhibitor. This co-ingestant may have affected renal function and the ability to excrete potassium. Lisinopril may also have contributed to the prolonged period of hypotension. We discuss the role of dialysis in the management of potassium overdose based on our analysis of this case and the few other cases reported in the medical literature. 


\section{Case Report}

In a suicide attempt, a 56-year-old female drank a slurry created in a blender consisting of an unknown amount of her $10 \mathrm{mg}$ lisinopril and $20 \mathrm{mEq}$ extended release potassium tablets. After a delay of several hours, she called an ambulance, and arrived in the emergency department (ED) $5 \mathrm{~h}$ post-ingestion. An examination of the empty pill bottles revealed that the potassium prescription had been filled 4 days prior to presentation with 120 tablets. The lisinopril prescription had been filled 4 days prior to presentation with 60 tablets. She had a past history of hypertension and depression. She was also on fluoxetine, but this was reportedly not ingested. There was no past history of drug or alcohol abuse.

During ambulance transport, the patient was reported to be lethargic and bradycardic with a heart rate between 30 and 40 beats per minute, with a palpable pulse. She received no specific therapies during transport. On arrival in the ED, the patient had agonal respirations, and was pulseless and unresponsive to all stimuli. The cardiac rhythm revealed a wide complex configuration consistent with a "sine wave", with a rate between 30 and 40 beats per minute. (Note the initial 12-lead electrocardiogram in Fig. 1).

Given the history of potassium ingestion, the patient received $2 \mathrm{~g}$ of calcium gluconate, $50 \mathrm{~g}$ of dextrose, 5 units of regular insulin, and $100 \mathrm{mEq}$ of sodium bicarbonate. These medications were administered intravenously. Initial resuscitative measures also included endotracheal intubation, chest compressions, and intravenous fluid administration. The cardiac rhythm deteriorated to ventricular fibrillation and ventricular tachycardia on several occasions, but responded to defibrillation. Transcutaneous and transvenous pacing were attempted with limited ability to capture. The patient's initial 2-h course remained critical with labile blood pressures in the range of 70 to $90 \mathrm{mmHg}$ systolic. Within the first hour after presentation, gastric lavage was performed with return of multiple pill fragments, and activated charcoal was administered. The rationale for performing gastrointestinal decontamination over $5 \mathrm{~h}$ after ingestion was because of possible inaccuracies in the history.

Two hours after arrival in the ED, the patient was in a wide complex cardiac rhythm. Systolic blood pressure in the 1970s was maintained with the use of $20 \mu \mathrm{g} \mathrm{kg}^{-1} \mathrm{~min}^{-1}$ of intravenous dopamine and $5 \mu \mathrm{g} / \mathrm{min}$ of intravenous epinephrine. Cardiopulmonary examination revealed clear breath sounds bilaterally and a regular rate and rhythm with no murmur, rub, or extra heart sounds. The abdomen was soft with no tenderness to palpation and no organomegaly. Neurological examination revealed no response to verbal or painful stimuli. There was no extremity edema.

Laboratory values from initial presentation revealed a serum sodium concentration of $140 \mathrm{mEq} / \mathrm{L}$, potassium $10.2 \mathrm{mEq} / \mathrm{L}$, chloride $116 \mathrm{mEq} / \mathrm{L}, \mathrm{BUN} 20 \mathrm{mg} / \mathrm{dL}$, and creatinine $1.3 \mathrm{mg} / \mathrm{dL}$. Toxicology screening of urine was negative for cocaine, cannabinoids, amphetamine, barbiturates, benzodiazepines, opiates, phencyclidine, and methadone. Serum analysis revealed an ethanol concentration of $0 \mathrm{mg} / \mathrm{dL}$, acetaminophen $3.1 \mu \mathrm{g} / \mathrm{mL}$, and salicylate $1.7 \mathrm{mg} / \mathrm{dL}$. Toxicologic analysis for lisinopril and other antihypertensive agents was not performed. Approximately $1 \mathrm{~h}$ into the resuscitation, the serum potassium was $11 \mathrm{mEq} / \mathrm{L}$ despite the medications used to treat hyperkalemia. Two hours after ED presentation, an arterial blood gas on $100 \%$ oxygen revealed a $\mathrm{pH}$ of 7.12, $\mathrm{pCO}_{2} 34.0 \mathrm{mmHg}$, and $\mathrm{pO}_{2} 434.0 \mathrm{mmHg}$.

A cardiologist was consulted on the case and performed an echocardiogram in the ED which revealed normal cardiac function (ejection fraction not specified). A nephrology consult was obtained for emergent hemodialysis due to persistent hyperkalemia and cardiac arrest. Early in the patient's ED course, the nephrologist reasoned that with normal renal function, the potassium would be excreted rapidly, and hemodialysis was not needed. Given the prolonged period of hemodynamic instability, and after noting a decrease in urine output, hemodialysis was initiated approximately $4 \mathrm{~h}$ after presentation (9-h post-ingestion).
Fig. 1 Electrocardiogram on arrival to ED demonstrating wide complex "sine wave" rhythm consistent with hyperkalemia

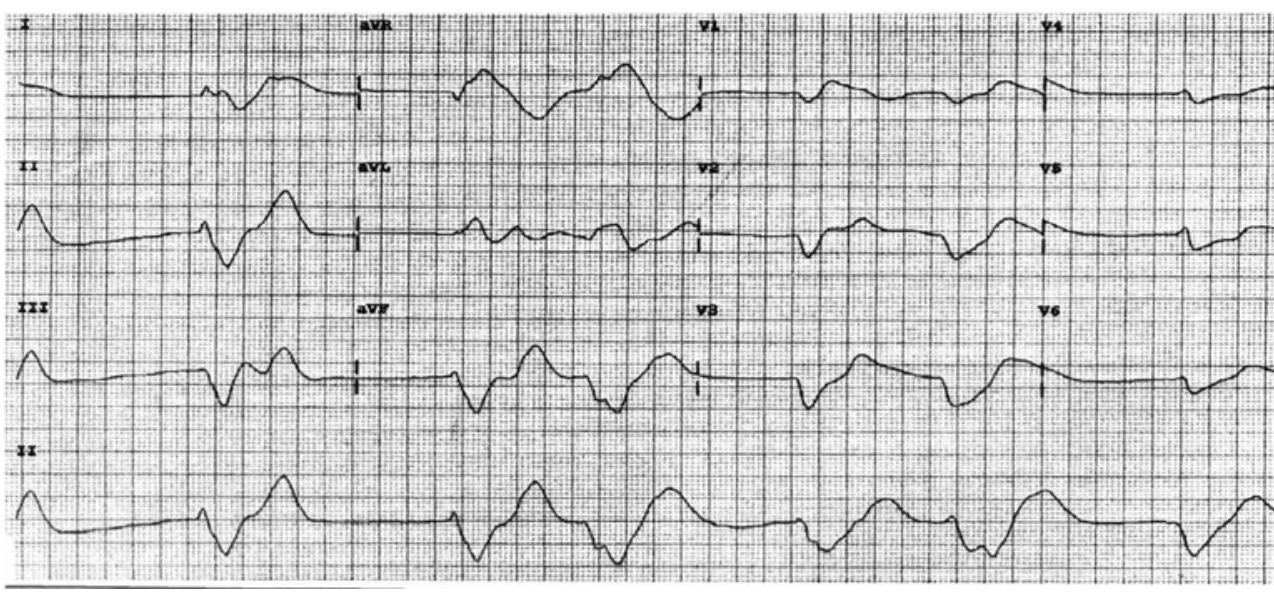


The patient was given two $30 \mathrm{~g}$ doses of sodium polystyrene sulfonate (kayexalate ${ }^{\circledR}$ ), $2 \mathrm{~h}$ apart, via nasogastric tube during dialysis. The serum potassium concentration was $3.5 \mathrm{mEq} / \mathrm{L} 30 \mathrm{~min}$ after dialysis. The patient's heart rhythm return to a normal sinus rhythm, but vasopressor support was still required. A 12-lead electrocardiogram (noted in Fig. 2) was obtained after dialysis (approximately $16 \mathrm{~h}$ after presentation to the $\mathrm{ED}$ ).

No further doses of sodium polystyrene sulfate were administered after initial dialysis. On hospital day 3, the serum potassium concentration had gradually risen to $7.7 \mathrm{mEq} / \mathrm{L}$, and the patient underwent hemodialysis again. The serum potassium concentration remained in a normal range throughout the remainder of her hospital stay. By hospital day 5 , blood pressures rose to a normal range and the patient was weaned from dopamine and epinephrine. On hospital day 7, the patient was noted to have symptoms of depression, but the neurologic examination was unremarkable, and she was transferred to an inpatient psychiatric facility. At discharge, serum analysis revealed a BUN concentration of $18 \mathrm{mg} / \mathrm{dL}$ and creatinine $1.2 \mathrm{mg} / \mathrm{dL}$.

\section{Discussion}

Hyperkalemia due to acute potassium overdose is uncommon. There are no large series of potassium overdose cases in the medical literature. The patient described in this report also ingested an ACE inhibitor, which may have contributed to the elevated potassium concentration. Although not suspected in this case, other toxins that can cause hyperkalemia, by various mechanisms, include angiotensinreceptor blockers, beta-adrenergic antagonists, digoxin, nonsteroidal anti-inflammatory drugs, spironolactone, and succinylcholine.

ACE inhibitors suppress aldosterone production, which results in increased sodium excretion and reduced potassium and hydrogen ion excretion. ACE inhibitors also may cause hypotension in the overdose setting, leading to decreased renal perfusion and function [1]. Significant toxicity due to ACE inhibitor overdose is unusual.

In the case presented here, the initial serum creatinine concentration of $1.3 \mathrm{mg} / \mathrm{dL}$ is in the high normal range. Although glomerular filtration rate was not calculated, some degree of renal dysfunction may have contributed to decreased ability of the kidneys to excrete potassium and lisinopril. Lisinopril may have contributed to prolonged hypotension, as hyperkalemia alone could not explain this. However, it appears unlikely that the 5-day period of hypotension could have been caused solely by lisinopril. Pharmacokinetic data in lisinopril overdose are limited. In one case of overdose, the half-life was $14.9 \mathrm{~h}$ and the half-life is $12 \mathrm{~h}$ when taken in therapeutic doses [2]. In our case, there may have been other toxins present that contributed to hypotension that were not tested for. Given the normal echocardiogram in the ED, it is unlikely that an acute cardiomyopathy could have explained the prolonged hypotension.

There is limited discussion in the medical literature of indications for dialysis after acute potassium overdose. Potassium is one of the relatively few toxins that is easily dialyzed based on its water solubility, small size, and limited protein binding [3]. Potassium removal is slow with peritoneal dialysis; hemodialysis is the preferred extracorporeal removal method.

Review of the medical literature reveals good outcomes in cases that were dialyzed versus both good and bad outcomes in those that were not. The most striking example of a case where dialysis was not employed was in a 6-week-old infant who had undergone surgical repair of truncus arteriosus and had been given a parenteral antibiotic that was erroneously prepared with potassium chloride rather than a glucose solution. She developed cardiac arrest with a "severe arrhythmia" (not specified in the report), and was found to have a potassium concentration of $17.7 \mathrm{mEq} / \mathrm{L}$. Renal

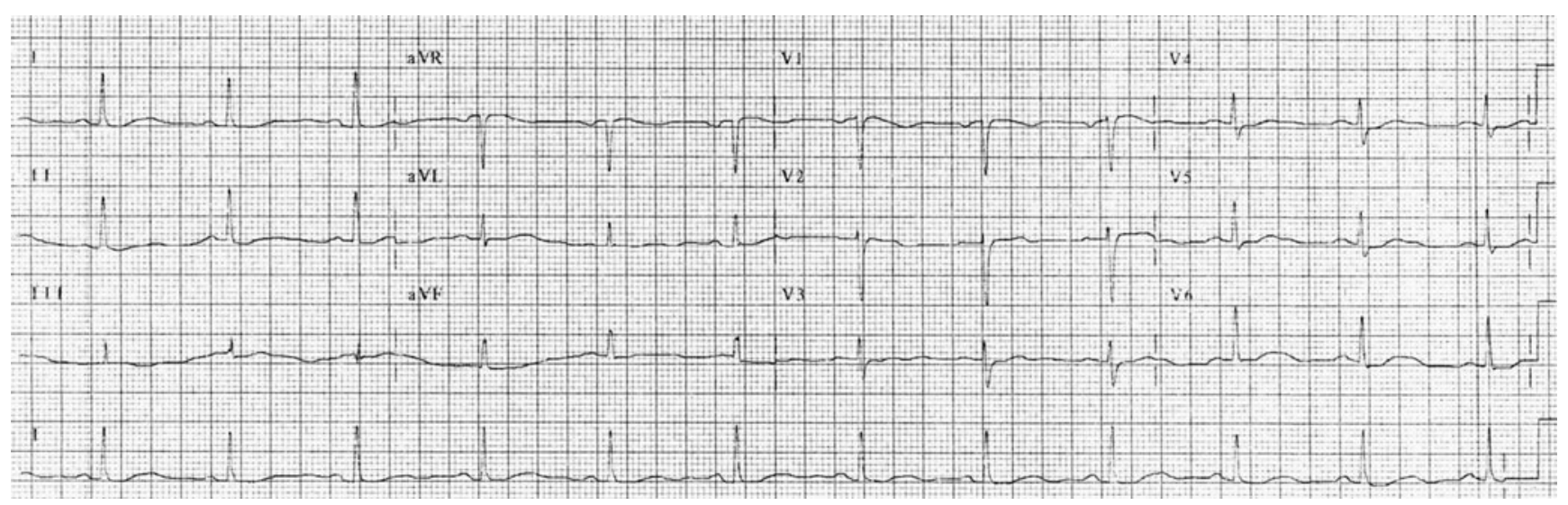

Fig. 2 Electrocardiogram demonstrating narrow complex rhythm with absence of peaked T waves after hemodialysis and normalization of serum potassium (16 $\mathrm{h}$ after presentation to the ED) 
function is not noted in the report, and dialysis was not undertaken due to her small size and extensive thromboses in the major venous vessels. She survived, and on follow-up at the age of 4 years, the patient was neurologically intact except for severe iatrogenic hearing loss [4].

There are other cases in the literature in which potassium levels were elevated, with good outcomes after supportive care, but dialysis was not performed. A 36-year-old male took an overdose of slow-release potassium chloride and presented with a "non-sine wave" wide QRS complex rhythm, peaked $\mathrm{T}$ waves, short runs of ventricular tachycardia, and a potassium concentration of $8.9 \mathrm{mEq} / \mathrm{L}$, with no documentation of renal function. Treatment consisted primarily of intravenous glucose, insulin, sodium bicarbonate, and calcium [5]. Ingestion of a salt substitute in an 8-monthold resulted in a "sine wave" rhythm, with a potassium concentration of $10.9 \mathrm{mEq} / \mathrm{L}$, creatinine $0.6 \mathrm{mg} / \mathrm{dL}$, and good outcome without dialysis [6]. A 27-year-old female took an overdose of sustained-release potassium chloride and presented with a "non-sine wave" wide complex QRS rhythm, peaked $\mathrm{T}$ waves, a potassium concentration of $9.1 \mathrm{mEq} / \mathrm{L}$, and blood urea nitrogen $9.5 \mathrm{mg} / \mathrm{dL}$ [7]. She too had full recovery without dialysis.

Bad outcomes with similarly elevated potassium concentrations and medical management without dialysis are also reported. A 46-year-old female was brought to an emergency department in cardiac arrest $1 \mathrm{~h}$ after an overdose of slow-release potassium tablets. Initial cardiac rhythms included "sine wave" and ventricular fibrillation, and the potassium concentration was $9.6 \mathrm{mEq} / \mathrm{L}$. Renal function is not noted in the report. Stabilization measures included defibrillation on three attempts, as well as intravenous calcium, glucose, insulin, and sodium bicarbonate. Sinus rhythm was restored and potassium concentrations returned to normal, but the patient died after remaining in a persistent comatose state for 14 days [8]. Early dialysis would not have been possible in this case, as she presented in cardiac arrest.

A 29-year-old female ingested salt substitute and presented with a wide complex QRS rhythm (morphology not delineated) and peaked $\mathrm{T}$ waves, with a potassium concentration of $8.4 \mathrm{mEq} / \mathrm{L}$. Serum creatinine was $1.2 \mathrm{mg} / \mathrm{dL}$. At the time the lab values returned, she suffered a cardiac arrest which was treated with standard resuscitative measures, as well as intravenous calcium, glucose, insulin, and sodium bicarbonate. Normokalemia was restored after $3 \mathrm{~h}$, and the cardiac rhythm was stabilized. She developed post-hypoxic encephalopathy and quadriplegia, and was transferred to care in another country 24 days post-presentation [9]. It is unclear as to what the outcome would have been had potassium lowering medications been administered at the time of presentation. Dialysis would not have been possible at the time of arrest, and did not appear justified after stabilization as the potassium concentration dropped rapidly over a period of $3 \mathrm{~h}$.

Hemodialysis was utilized in a case of a sustainedrelease potassium chloride overdose in a 50-year-old female. The patient presented with normal renal function (creatinine $0.6 \mathrm{mg} / \mathrm{dL}$ ), a potassium concentration of $8.2 \mathrm{mEq} / \mathrm{L}$, and peaked $\mathrm{T}$ waves without $\mathrm{QRS}$ widening on the electrocardiogram. Treatments included intravenous calcium, sodium bicarbonate, glucose, and insulin. Transient "sine wave" rhythm changes responded to intravenous boluses of calcium and sodium bicarbonate. Serum potassium concentrations were still elevated at 7.7 and $9.0 \mathrm{mEq} / \mathrm{L}$ at 3 and $7 \mathrm{~h}$ after presentation, respectively. Hemodialysis was subsequently initiated, and the patient was discharged to home after several days (not specified) [10].

Another case in which hemodialysis was undertaken involved a 54-year-old female who ingested salt substitute. She presented to an ED with wide complex "sine wave" pulseless electrical activity. Resuscitative measures including empiric treatment of hyperkalemia with medications were initiated. The initial potassium concentration was $8.2 \mathrm{mEq} / \mathrm{L}$, with a creatinine of $2.1 \mathrm{mg} / \mathrm{dL}$. Sinus rhythm with systolic blood pressure over $100 \mathrm{mmHg}$ was restored. The patient was dialyzed and was discharged to home after a 1-week hospital stay with minimal short-term memory impairment [11].

Based on these cases, it is clear that potassium overdose with significant hyperkalemia and a wide complex QRS complex rhythm can lead to both good and bad outcomes without dialysis. The two cases described above, as well as the case described in this report, had good outcomes with dialysis. One of the two cases from the literature had renal dysfunction, and our case had a creatinine in the high normal range. The decision to dialyze is a judgment based on the degree of hyperkalemia, clinical state, electrocardiographic analysis, renal function, and availability of dialysis itself. Hemodialysis should be considered in cases of potassium overdose with significant hyperkalemia and underlying renal dysfunction. If renal function is normal, we recommend hemodialysis in the setting of cardiovascular instability, unless medical therapy results in immediate improvement in clinical state and a rapid decline in serum potassium concentrations. Unfortunately, dialysis is difficult to undertake in a patient with cardiovascular instability, and impossible in the setting of cardiac arrest. In stable patients with normal renal function, potassium excretion would likely be rapid.

The patient in this report had a delayed rise in potassium concentration on hospital day 3. This phenomenon was most likely due to delayed absorption of the sustainedrelease preparation, although redistribution effects are theoretically possible. Lisinopril may have also played a role, due to its effect on renal perfusion and function. 
Patients who overdose on sustained-release potassium preparations need to be monitored for delayed onset of hyperkalemia, and like the patient in this report, hemodialysis may be necessary.

Limitations of this report include the difficulty in making recommendations based on a retrospective case review. Conclusions regarding the prolonged period of hypotension are also difficult to make as testing for lisinopril and other antihypertensive agents was not done.

\section{Conclusion}

This was a case of intentional potassium overdose resulting in significant hyperkalemia and hemodynamic instability, with a good outcome after utilizing both potassium lowering medications and hemodialysis. As oral potassium overdoses are uncommon, the role of dialysis, especially in the setting of normal renal function, is not clearly delineated in the medical literature. We recommend hemodialysis in cases of potassium overdose with significant hyperkalemia and cardiovascular instability, unless pharmacologic management results in rapid clinical improvement and decline of potassium concentration. Hemodialysis should also be considered in patients with hyperkalemia and renal insufficiency.

\section{References}

1. Lip GYH, Ferner RE (1995) Poisoning with anti-hypertensive drugs: angiotensin converting enzyme inhibitors. J Hum Hypertens 9:711-715

2. Dawson AH, Harvey D, Smith AJ et al (1990) Lisinopril overdose [letter]. Lancet 35:487-488

3. Goldfarb DS, Matalon D (2006) Principles and techniques applied to enhance elimination. In: Flomenbaum NE, Goldfrank LR, Hoffman RS, Howland MA, Lewin NA, Nelson LS (eds) Goldfrank's Toxicologic Emergencies, 8th edn. McGraw-Hill, New York

4. Horisberger T, Fischer JE, Waldvogel K (2004) Long-term outcome of an infant resuscitated from iatrogenic potassium intoxication with a serum level of $17.7 \mathrm{mmol} / \mathrm{L}$. Eur J Pediatr 163:48-49

5. Illingworth RN, Proudfoot AT (1980) Rapid poisoning with slowrelease potassium. Br Med J 281:485-486

6. Kallen RJ, Rieger CHL, Cohen HS et al (1976) Near-fatal hyperkalemia due to ingestion of salt substitute by an infant. JAMA 235:2125-2126

7. Steedman DJ (1988) Poisoning with sustained-release potassium. Arch Emerg Med 5:206-211

8. Saxena K (1988) Death from potassium chloride overdose. Postgrad Med 84:97-102

9. Schim van der Loeff HJ, Strack van Schijndel RJM, Thijs LG (1988) Cardiac arrest due to oral potassium intake. Intensive Care Med 15:58-59

10. Su M, Stork C, Ravuri S et al (2001) Sustained-release potassium chloride overdose. Clin Toxicol 39:641-648

11. Pang PS, Look RB, Brown DFM, Nadel ES (2004) Wide complex rhythm and cardiac arrest. J Emerg Med 26:197-200 